\title{
Special issue on multimedia presentation assisted clinical diagnosis, prognosis and treatment (II)
}

\author{
Preface by the Guest Editor:
}

To follow our successful Special Issue on Multimedia Presentation Assisted Clinical Diagnosis, Prognosis and Treatment [1], this issue of the Journal of X-ray Science and Technology has continued to be well represented with seven multimedia assisted clinical papers. These include, for example, conebeam CT in dental diseases in evaluation of a serial of rare cases, including maxillofacial fractures, ameloblastomas of lower jaw and lymphoeithelial carcinoma in parotid gland. In addition, in evaluation of 3D reconstruction of CT complicated hydronephrosis caused by aberrant renal vessel, rosai-dorfman disease with kidney involvement, snakebite-induced cerebral infarction and pediatric malignant bone tumors. The rotated multimedia 3D videos provide teaching tools, research approach or precision diagnostic and treatment aid.

As guest editor of this special issue, I would like to take this opportunity to thank the Advisory Editor in Chief and Professor Hong Liu for his visionary leadership to the journal, and to introduce 3D videos in clinical reports. I am also grateful for the patience and assistance provided by the Editor-in-Chief and Professor Bin Zhen. Without their contribution this special issue would not have been possible. Finally, I have to thank authors for all their efforts in preparing these papers and to thank the reviewers for their reconstructive comments and valuable contribution to ensure that a high standard and good quality can be retained.

Yuanyuan Zhang, MD, Ph.D.

\section{Reference}

[1] H. Liu, and Y. Zhang, Introduction and Preface, Multimedia presentation assisted clinical diagnosis, prognosis and treatment, J Xray Sci Technol 24(5) (2016), 647. 